\title{
L. Dade Lunsford and Jason P. Sheehan (eds): Intracranial stereotactic radiosurgery
}

\author{
Thieme Medical Publishers, New York 2009, ISBN 978-1-60406-200-7
}

\author{
Vincenzo Cuccurullo
}

Published online: 17 December 2009

(C) Springer-Verlag 2009

This book is edited by L. Dade Lunsford and Jason P. Sheehan, two recognized international experts in the field of neurosciences, with reference to imaging, surgery and radiotherapy. It is a very specialised publication, therefore mainly directed to radiotherapists, but can also be interesting for surgeons and experts in neuroimaging who want to learn more about complementary indications for this approach or better understand how to define a target or monitor the disease.

The major aim of the book is to provide instruction in the current practice of intracranial stereotactic radiosurgery, a field that continues to expand and mature. To reach their goal the authors tried to give an important source of information for those who wish to improve their knowledge in this field of interest. The value of the publication is strongly increased by the small number of recent books discussing this topic and by the updated bibliography. Moreover, the images are of good quality and effective in clarifying most of the important issues connected with stereotactic radiosurgery.

The book is organized into 20 short chapters: (1) from notion to practice, (2) radiobiology, (3) how brain tumours respond, (4) image-guided radiosurgery, (5) stereotactic radiosurgery for arteriovenous malformations, (6) stereotactic radiosurgery for fistulas, (7) stereotactic radiosurgery for cavernomas, (8) stereotactic radiosurgery for meningi- omas, (9) stereotactic radiosurgery for pituitary adenomas, (10) stereotactic radiosurgery for chordomas and chondrosarcomas, (11) stereotactic radiosurgery for glomus tumours, (12) stereotactic radiosurgery for vestibular schwannomas, (13) stereotactic radiosurgery for non-vestibular schwannomas, (14) stereotactic radiosurgery for trigeminal neuralgia, (15) stereotactic radiosurgery for movement disorders, (16) stereotactic radiosurgery for epilepsy, (17) stereotactic radiosurgery for ocular disorders, (18) stereotactic radiosurgery for gliomas, (19) stereotactic radiosurgery for brain metastases and (20) stereotactic radiosurgery for paediatric brain tumours.

As can be observed, after a general introduction in the first four chapters, the following are devoted to specific issues. Each chapter includes a brief but detailed discussion on indication, methods, outcomes and complications associated with radiosurgery.

The fundamentals on stereotactic radiosurgery are explained in great detail, with accuracy and precision. The most important concepts are repeated and stressed at the relevant junctures throughout the book.

The combined efforts of the editors and contributing authors have produced this simple and sharp book that will be useful to surgeons, radiation oncologists and residents approaching the fast-evolving field of intracranial stereotactic radiosurgery.

V. Cuccurullo $(\bowtie)$

Medicina Nucleare, Seconda Università di Napoli, Naples, Italy

e-mail: vincenzo.cuccurullo@unina2.it 